\title{
Metaplectic sheets and caustic traversals in the Weyl representation
}

\author{
Alfredo M Ozorio de Almeida ${ }^{1}$ and Gert-Ludwig Ingold ${ }^{2}$ \\ ${ }^{1}$ Centro Brasileiro de Pesquisas Fisicas, Rua Xavier Sigaud 150, 22290-180, Rio \\ de Janeiro, R.J., Brazil \\ ${ }^{2}$ Institut für Physik, Universität Augsburg, Universitätstraße 1, D-86135 \\ Augsburg, Germany \\ E-mail: ozorio@cbpf.br and gert.ingold@physik.uni-augsburg.de
}

\begin{abstract}
The quantum Hamiltonian generates in time a family of evolution operators. Continuity of this family holds within any choice of representation and, in particular, for the Weyl propagator, even though its simplest semiclassical approximation may develop caustic singularities. The phase jumps of the Weyl propagator across caustics have not been previously determined.

The semiclassical appproximation relies on individual classical trajectories together with their neighbouring tangent map. Based on the latter, one defines a continuous family of unitary tangent propagators, with an exact Weyl representation that is close to the full semiclassical approximation in an appropriate neighbourhood. The phase increment of the semiclassical Weyl propagator, as a caustic is crossed, is derived from the facts that the corresponding family of tangent operators belong to the metaplectic group and that the products of the tangent propagators are obtained from Gaussian integrals. The Weyl representation of the metaplectic group is here presented, with the correct phases determined within an intrinsic ambiguity for the overall sign. The elements that fully determine the phase increment across a particular caustic are then analysed.
\end{abstract}

PACS numbers: 03.65.-w, 03.65.Sq 


\section{Introduction}

The construction of a path integral for the Weyl propagator 1, 2, that is, the evolution operator, $\widehat{U}_{t}=\exp (-\mathrm{i} t \widehat{H} / \hbar)$, in the Weyl representation, makes no restriction on the form of the Hamiltonian that generates the continuous evolution of a quantum system. Except for possible minor modifications due to ordering, the Weyl symbol, $H(\mathbf{x})$, for the quantum Hamiltonian, $\widehat{H}$, is identified with its classical counterpart, $H(\boldsymbol{x})$, where $\{\boldsymbol{x}=(\mathbf{p}, \mathbf{q})\}$ is the $2 N$-dimensional classical phase space, $\mathbf{R}^{2 N}$. There is no need for the classical Hamiltonian to be derived from a Lagrangian, so that the path integral may just as easily be constructed for e.g. the Kerr Hamiltonian [3, 4, 5, $H(\boldsymbol{x})=\left(a \mathbf{p}^{2}+b \mathbf{q}^{2}\right)^{2}$, as for the usual form, $H(\boldsymbol{x})=\mathbf{p}^{2} / 2 m+V(\mathbf{q})$.

A classical trajectory coincides with a stationary phase of the Weyl path integrand, just as with Feynman path integrals in the position representation. Thus, one obtains semiclassical (SC) approximations for the full path integral as a superposition of a few contributing classical trajectories. The main difference is that here the relevant trajectory is prescribed by a kind of boundary condition on the centre, $\mathbf{x} \equiv\left(\boldsymbol{x}^{+}+\boldsymbol{x}^{-}\right) / 2$, of its pair of endpoints, $\boldsymbol{x}^{-}$and $\boldsymbol{x}^{+}$, rather than by the pair of end-positions $\left(\mathbf{q}^{-}, \mathbf{q}^{+}\right)$[2, 6]. In both the Weyl and the position representations, contributing trajectories may coalesce, with the passage of time, or with continuous change in the boundary condition. At these caustics, the SC approximation has a singularity. In passing a caustic, SC approximations generally change their phase by $\mu \pi / 2$, where $\mu$ is known as the Maslov index [7, 8]. If $H(\boldsymbol{x})=\mathbf{p}^{2} / 2 m+V(\mathbf{q})$, then, in the position representation, $\mu$ coincides with the Morse index [9]. A more general geometrical phase space treatment of the Maslov index for periodic orbits is presented in references [10, 11].

The semiclassical approximation becomes exact in the limit where the Hamiltonian is quadratic in the phase space variables. Classically, such a Hamiltonian generates a (linear) symplectic transformation between the endpoints of a classical trajectory: $\boldsymbol{x}^{-} \mapsto \boldsymbol{x}^{+}=\mathbf{M} \boldsymbol{x}^{-}$, where $\mathbf{M}$ is a symplectic matrix. The symplectic subgroup of classical canonical transformations, $\operatorname{Sp}(2 N)$, characterized by $\mathbf{M}$, corresponds to the metaplectic subgroup, $\operatorname{Mp}(2 N)$, of general quantum unitary transformations, $\widehat{U}_{\mathbf{M}}$, that is, the group of metaplectic operators [12, 13, 14, 15, 16, 17, 18. The amplitude of the Weyl propagator for a metaplectic operator is constant throughout phase space, but it has a true caustic singularity at an instant when the $\mathrm{SC}$ form of the Weyl propagator must be replaced by a Dirac delta-function.

The action of a Weyl propagator on other operators involves phase space integrals. Therefore, in the general SC scenario where the region of integration may encompass different Maslov phases, it is crucial that these be correctly evaluated. Indeed, such switches of phase can be more important than the possibly integrable SC singularities at the caustics themselves, as occurs with the initial value or final value algorithms recently proposed in [19. Furthermore, there are applications where SC super propagators for Wigner functions [20, 21] are specified by products of Weyl propagators, each of which may traverse its own caustics [19, 22, so that it is indispensible to determine the correct Maslov phases in all cases. The purpose of the present paper is to establish the general connections of SC Weyl propagators (and of their Fourier transforms) through their caustics.

In order to establish possible phase changes for caustic traversals, one needs to focus on the tangent map for the full canonical transformation in the neighbourhood of a given trajectory. In other words, one must study the symplectic approximation 
to the full canonical transformation between the neighbourhoods of the endpoints of a classical trajectory: $\delta \boldsymbol{x}^{-} \mapsto \delta \boldsymbol{x}^{+}=\mathbf{M}_{\mathbf{x}} \delta \boldsymbol{x}^{-}$, where the symplectic matrix for the transformations is labelled by the centre, $\mathrm{x}=\left(\boldsymbol{x}^{+}+\boldsymbol{x}^{-}\right) / 2$, of the main trajectory. This is an essential ingredient of the SC approximation of the Weyl propagator, $U(\mathbf{x})$. Furthermore, one can also construct an exact metaplectic tangent operator, $\widehat{U}_{\mathbf{M}_{\mathbf{x}}}$, represented by the Weyl symbol $U_{\mathbf{M}_{\mathbf{x}}}\left(\mathbf{x}^{\prime}\right)$, i.e. the tangent Weyl propagator, based on the same symplectic matrix, $\mathbf{M}_{\mathbf{x}}$. Just as the tangent map is close to the full canonical transformation in the neighbourhood of its endpoints, we can define a small neighbourhood $\delta \mathbf{x}^{\prime}$, to be measured from the centre, $\mathbf{x}$, and then we have $U_{\mathbf{M}_{\mathbf{x}}}\left(\delta \mathbf{x}^{\prime}\right) \approx U_{S C}\left(\delta \mathbf{x}^{\prime}\right)$. $\mathbf{t}$ It is important to emphasize that this is a purely local approximation and that different arguments of the Weyl propagator (i.e. choices of the centre, $\mathbf{x}$ ) are related to different trajectories with different linearized maps, so that the pure quantum tangent propagator is constructed entirely from a local classical map. Hence, one can investigate the possible phase jumps of the SC approximation of the Weyl propagator by analyzing, instead, the behaviour of the exact symbol for the tangent propagator.

The tangent Weyl propagators belong to the metaplectic group. The difficulty is that, even though $\operatorname{Sp}(2 N)$ and $\operatorname{Mp}(2 N)$ are isomorphic in the neighbourhood of the identity, the correspondence $\mathbf{M} \mapsto \widehat{U}_{\mathbf{M}}$ is not one to one: There is a double covering of the quantum group, i.e. a pair of metaplectic operators and, hence, a pair of metaplectic Weyl propagators for each $\mathbf{M}$ : $\widehat{U}_{\mathbf{M}}^{-}=-\widehat{U}_{\mathbf{M}}^{+}$. In particular, one should note that important particular symplectic matrices, such as $\mathbf{M}=\mathbf{I}$, the identity matrix, and $\mathbf{M}=-\mathbf{I}$, the reflection at the origin, correspond to pairs of metaplectic operators, even though only one of these is commonly employed. Since they only differ by an overall sign, the choice of different sheets of a metaplectic operator has no essential effect on how it acts on wave functions and this action completely cancels out for Heisenberg operator evolution: $\widehat{A} \mapsto \widehat{U}^{\dagger} \widehat{A} \widehat{U}$. Nonetheless, the choice of sheet is essential for SC applications and it lies in the focus of this paper, but we shall distinguish the sheet in the notation for $\widehat{U}_{\mathbf{M}}$ only where it is essential.

The notion of crossing a caustic needs to be carefully dissected in terms of corresponding families of classical maps and quantum operators: The quantum Hamiltonian generates a continuous one-parameter family of evolution operators, $\widehat{U}_{t}$, corresponding semiclassically to a family of canonical transformations, each of which may, in turn, be decomposed into its individual classical trajectories. In the neighbourhood of each of these trajectories, one can then define a family of tangent symplectic maps, $\mathbf{M}_{t}$, and hence, a continuous one-parameter family of tangent metaplectic operators: $\widehat{U}_{\mathbf{M}_{t}}$. 国 Such a family does not form a subgroup of the metaplectic operators, because it is not closed with respect to multiplication, but the product rule for each pair of sequential time intervals is satisfied: For $t_{1}+t_{2}=t$, one has the exact product $\widehat{U}_{\mathbf{M}_{t_{2}}} \widehat{U}_{\mathbf{M}_{t_{1}}}=\widehat{U}_{\mathbf{M}_{t}}$, corresponding to symplectic matrices, which also satisfy $\mathbf{M}_{t_{2}} \mathbf{M}_{t_{1}}=\mathbf{M}_{t}$, even though neither $\left(\mathbf{M}_{t}\right)^{2}$ nor $\left(\widehat{U}_{\mathbf{M}_{t}}\right)^{2}$ need belong

$\dagger$ One should recall that the Weyl representation is invariant with respect to phase space translations, so that the change of origin in the argument, $\mathbf{x}^{\prime} \mapsto\left(\delta \mathbf{x}^{\prime}=\mathbf{x}^{\prime}-\mathbf{x}\right)$, of the SC approximation to $U\left(\mathbf{x}^{\prime}\right)$ is purely classical.

$\ddagger$ Strictly, one should define $\mathbf{M}_{\mathbf{x}(t)}$ rather than $\mathbf{M}_{t}$ following the previous notation, because the centre, $\mathbf{x}(t)$, of a given trajectory with fixed initial condition is itself time dependent. Indeed, one also deals with caustics as the centre $\mathbf{x}$ is varied while the time is fixed, but one needs to consider only one-parameter families of metaplectic operators, so this has been labelled as $t$ thoughout this paper. 
to their respective family.

Continuity of the classical family of symplectic transformations entails the continuity of the family of tangent operators, but this crucial point will be masked by caustics in any given representation. A caustic of the tangent Weyl propagator occurs for the same matrix $\mathbf{M}_{t}$ as for the SC approximation of the full evolution operator $\widehat{U}_{t}$, but it is a true singularity, at which the Weyl symbol becomes a $\delta$-function [17. The fact that there is only a pair of metaplectic sheets, corresponding to the choice of a $\pi$ phase (i.e. an overall sign) for the Weyl propagator, does not prevent a possible phase change of $\pm \pi / 2$ across a caustic. However, it will be shown that it is only the overall sign that needs to be determined by the history of each trajectory, because otherwise factors of i for tangent propagators can be inferred from the dynamical properties of the symplectic matrix $\mathbf{M}_{t}$.

Fortunately, the caustics of different representations occur for different values of the trace of $\mathbf{M}_{t}$ and thus at different parameter values $t$. This allows for swapping representations, where the continuity in a $t$-interval for one representation supplies the sign change at the caustic of the other. An alternative method to evaluate the overall sign of the propagator beyond a caustic is based on the exact product for sequential time intervals. These can be chosen so that both propagators lie in the causticless neighbourhood, which guarantees that the generalized Gaussian integral for their product, which lies beyond the caustic, supplies the correct sign.

Nontrivial phase changes may in principle also occur for the product of finite metaplectic operators, or for their product with translation (Heisenberg) operators, or reflection (parity) operators. The specification of these phases is important for the $\mathrm{SC}$ treatment of operator and super operator evolutions, such as [19]. The case of parity operators demands special care because they have been defined with different phases in different contexts, namely the basis operator for the Weyl representation differs from the operators belonging to the metaplectic group that we consider here.

After a review of basic classical formulae for the Weyl propagators involving symmetric matrices and generating functions in section 2, the Weyl representation of metaplectic operators and its Fourier transform, the chord representation, are presented in section 3. The choices which are here made for the overall phases, that were ommited in 2 , is justified by the general consistency of the propagators on switching representation (section 4) on the one hand, and from their products (sections 5 and 6) on the other hand.

Given the elegance with which the double sheeted topology of the metaplectic group is here seen to be rendered within the Weyl representation, our aim is to provide a pragmatic recipe for determining the phase increment across each caustic, regardless of the metaplectic sheet. This is clearly synthetized in the concluding section 7 A didactical Appendix has been added to illustrate the theory in the quintessential example of products of harmonic oscilations.

\section{Classical ingredients}

The centre generating function, $S(\mathbf{x})$, defines implicitly a canonical transformation, $\boldsymbol{x}^{-} \mapsto \boldsymbol{x}^{+}$. This is achieved by a finite version of Hamilton's equations, that is, defining the chord, $\boldsymbol{\xi} \equiv \boldsymbol{x}^{+}-\boldsymbol{x}^{-}$, we have

$$
\boldsymbol{\xi}=-\mathbf{J} \frac{\partial S}{\partial \mathbf{x}}, \quad \text { or } \quad \mathbf{p}^{+}-\mathbf{p}^{-}=\boldsymbol{\xi}_{\mathbf{p}}=\frac{\partial S}{\partial \mathbf{q}}, \quad \mathbf{q}^{+}-\mathbf{q}^{-}=\boldsymbol{\xi}_{\mathbf{q}}=-\frac{\partial S}{\partial \mathbf{p}}
$$


where

$$
\mathbf{J}=\left(\begin{array}{cc}
0 & -1 \\
1 & 0
\end{array}\right)
$$

in terms of $(\mathbf{p}, \mathbf{q})$ blocks, that is, the standard symplectic matrix in Hamilton's equations. The endpoints are given by

$$
\boldsymbol{x}^{ \pm}=\mathrm{x} \pm \frac{\boldsymbol{\xi}}{2} .
$$

For the canonical transformation generated by a Hamiltonian during a short time, we thus have $S_{t}(\mathbf{x})=-t H(\mathbf{x})+\mathcal{O}\left(t^{3}\right)$, where the third order correction is given in 2].

The tangent map for the canonical transformation near a trajectory centred on $\mathbf{x}=\left(\boldsymbol{x}^{+}+\boldsymbol{x}^{-}\right) / 2$ is $\delta \boldsymbol{x}^{-} \mapsto \delta \boldsymbol{x}^{+}=\mathbf{M}_{\mathbf{x}} \delta \boldsymbol{x}^{-}$. It has the centre generating function corresponding to the symplectic matrix $\mathbf{M}_{\mathbf{x}}$

$$
S_{\mathbf{M}_{\mathbf{x}}}(\delta \mathbf{x})=\delta \mathbf{x} \cdot \mathbf{B}_{\mathbf{x}} \delta \mathbf{x},
$$

where $\mathbf{B}_{\mathbf{x}}$ is the (symmetric) Hessian matrix for the full generating function evaluated at $\mathbf{x}$. (Henceforth, the origin will be shifted so that $\delta \mathbf{x}=\mathbf{x}$.) Indeed, a symmetric matrix $\mathbf{B}$ defines the Cayley parametrization of a symplectic matrix $\mathbf{M}$ [2], and hence the symplectic transformation $\boldsymbol{x}^{+}=\mathbf{M} \boldsymbol{x}^{-}$, by

$$
\mathbf{M}=(\mathbf{I}+\mathbf{J B})^{-1}(\mathbf{I}-\mathbf{J B}),
$$

with the inverse

$$
\mathbf{J B}=(\mathbf{I}+\mathbf{M})^{-1}(\mathbf{I}-\mathbf{M}) .
$$

One should recall the fundamental symplectic property [23],

$$
\mathbf{M}^{\prime} \mathbf{J M}=\mathbf{J},
$$

where $\mathbf{M}^{\prime}$ is the transpose of $\mathbf{M}$.

It is important to note that any $2 N$-dimensional symmetric matrix, $\mathbf{B}$, defines a symplectic transformation through (2.1), unless $\mathbf{J B}$ has an eigenvalue -1 , whereas the condition (2.7) for the symplectic matrix $\mathbf{M}$ itself can only be verified a posteriori. The eigenvalues of $\mathbf{J B}$ must come in pairs, $\pm \gamma$ just as the eigenvalues of $\mathbf{J H}$, which determine the symplectic flow for the Hamiltonian, $H(\mathbf{x})=\mathbf{x} \cdot \mathbf{H x} / 2$. They correspond to the same eigenvectors for which $\mathbf{M}$ has a pair of eigenvalues $\left(\lambda, \lambda^{-1}\right)$. Again, the fact that the matrix $\mathbf{B}$ is real demands that any complex eigenvalues come in complex conjugate pairs (see e.g. 23]). Notwithstanding that $\operatorname{det}(\mathbf{B})=\operatorname{det}(\mathbf{J B})$, one should keep in mind that the eigenvalues of $\mathbf{B}$ are necessarily real, even if those of $\mathbf{J B}$ need not be.

For $N=1$, the transformation can be classified by $\operatorname{det}(\mathbf{B})$, that is, for $\operatorname{det} \mathbf{B}>0$ the transformation is elliptic, for $-1<\operatorname{det}(\mathbf{B})<0$ it is simply hyperbolic and for $\operatorname{det}(\mathbf{B})<-1$ hyperbolic with reflection. One should note that $\operatorname{det}(\mathbf{B})=\operatorname{det}(\mathbf{J B})=$ $\operatorname{tr}\left[(\mathbf{J B})^{2}\right] / 2$. The alternative classification of symplectic transformations in terms of

$$
\operatorname{tr}(\mathbf{M})=2 \frac{1-\operatorname{det}(\mathbf{B})}{1+\operatorname{det}(\mathbf{B})},
$$

or of $\operatorname{det}(\mathbf{B})$, is illustrated in figure 1 The classification, for $N=2$ according to the invariants of $\mathbf{J B}$ is given in 24] and, for larger dimensional phase spaces, a similarity transformation can always reduce the matrix $\mathbf{J B}$, just as the symplectic matrix $\mathbf{M}$, into 2-dimensional or 4-dimensional blocks [23, 25]. 


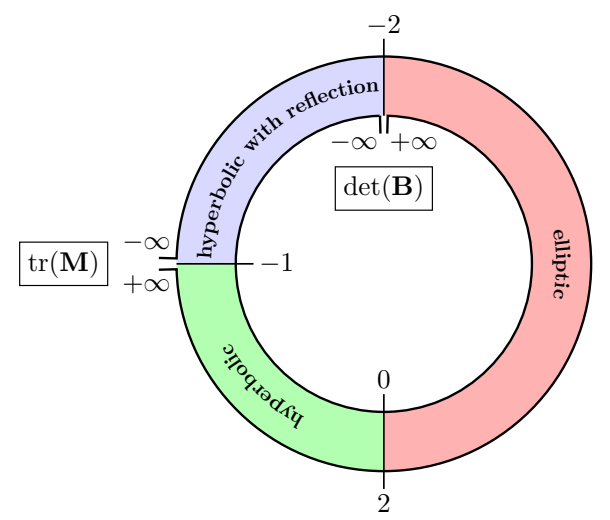

Figure 1. Classification of elliptic and hyperbolic symplectic motion for a single degree of freedom in terms of $\operatorname{tr}(\mathbf{M})$ or $\operatorname{det}(\mathbf{B})$.

A continuous transition between elliptic and hyperbolic transformations may pass through the identity, but, in general the boundary between them lies along the phase space shears, for which the normal form will be Cayley-parametrized by

$$
\mathbf{B} \rightarrow\left(\begin{array}{cc}
b & 0 \\
0 & 0
\end{array}\right), \mathbf{J B} \rightarrow\left(\begin{array}{cc}
0 & 0 \\
b & 0
\end{array}\right)
$$

for some real parameter $b$, such that $b=0$ for the identity. This is a parabolic transformation, such that its symplectic matrix has the form of a Jordan block:

$$
\mathbf{M} \rightarrow\left(\begin{array}{cc}
1 & 0 \\
-2 b & 1
\end{array}\right)
$$

The breakdown of the Cayley parametrization (2.5), such that $\operatorname{det}(\mathbf{B}) \rightarrow \pm \infty$, for a one-parameter family of transformations, if $N=1$, is specified by the limiting matrix,

$$
\mathbf{M} \rightarrow\left(\begin{array}{cc}
-1 & 2 b^{\prime} \\
0 & -1
\end{array}\right)
$$

so that the family of symplectic transformations goes through a shear with a reflection through the origin. This is the generic form for a caustic, i.e. for a single degree of freedom we have $\operatorname{tr}(\mathbf{M})=-2$, or equivalently, $\operatorname{det}(\mathbf{I}+\mathbf{M})=0$. But there is only one eigendirection, unless the parameter $b^{\prime}=0$ in (2.11), in which case the transformation reduces to a reflection at the origin.

The neighbourhood of a caustic can be neatly treated with the aid of the complementary Cayley parametrization [2, 18,

$$
\mathbf{M}=-(\mathbf{I}-\mathbf{J} \tilde{\mathbf{B}})^{-1}(\mathbf{I}+\mathbf{J} \tilde{\mathbf{B}})
$$

with its inverse:

$$
\mathbf{J} \tilde{\mathbf{B}}=-(\mathbf{I}-\mathbf{M})^{-1}(\mathbf{I}+\mathbf{M}) .
$$

From (2.5) and (2.12) one obtains the explicit expression for the new Cayley matrix as

$$
\tilde{\mathbf{B}}=-\mathbf{J B}^{-1} \mathbf{J}=\mathbf{J}^{-1} \mathbf{B}^{-1} \mathbf{J} .
$$

Thus, $\mathbf{B}^{-1}$ and $\tilde{\mathbf{B}}$ are related by a similarity transformation and can be diagonalized simultaneously, so that $\mathbf{B}$ and $\tilde{\mathbf{B}}$ have the same signature and their determinants 
have the same sign. It follows that $\operatorname{det}(\tilde{\mathbf{B}}) \rightarrow 0$ at a caustic, which allows for the neighbourhood of the caustic to be described by

$$
\tilde{\mathbf{B}} \rightarrow\left(\begin{array}{cc}
\tilde{b} & 0 \\
0 & 0
\end{array}\right), \mathbf{J} \tilde{\mathbf{B}} \rightarrow\left(\begin{array}{cc}
0 & 0 \\
\tilde{b} & 0
\end{array}\right),
$$

such that $\tilde{b}=0$ for a pure reflection. Thus, $\operatorname{det}(\mathbf{B})$ is small near the identity and diverges near the reflection, whereas the situation for $\operatorname{det}(\tilde{\mathbf{B}})$ is the reverse. Just as the Cayley matrix $\mathbf{B}$ specifies a quadratic centre generating function,

$$
\tilde{S}_{\mathbf{M}}(\boldsymbol{\xi})=\frac{1}{4} \boldsymbol{\xi} \cdot \tilde{\mathbf{B}} \boldsymbol{\xi}
$$

is the chord generating function for a symplectic transformation [2, such that $\mathbf{x}=$ $\mathbf{J} \partial \tilde{S} / \partial \boldsymbol{\xi}$ and again $\boldsymbol{x}^{ \pm}=\mathbf{x} \pm \boldsymbol{\xi} / 2$. Thus, whereas $S(\mathbf{x})$ becomes singular at a reflection, because all the chords then have the same centre, $\tilde{S}(\boldsymbol{\xi})$ becomes singular for a uniform translation, for which all centres have the same chord.

One should note that, since the eigenvalues of both $\mathbf{J B}$ and $\mathbf{J} \tilde{\mathbf{B}}$ come in pairs of opposite sign, it is generally true that $\operatorname{tr}(\mathbf{J B})=\operatorname{tr}(\mathbf{J} \tilde{\mathbf{B}})=0$. Furthermore, one obtains from (2.6) and (2.13) that the eigenvalues for $\mathbf{J B}$ and $\mathbf{J B}$ are related by $\tilde{\lambda}=-\lambda^{-1}$. Hence, $\operatorname{det}(\mathbf{B})$ and $\operatorname{det}(\tilde{\mathbf{B}})$ change signs simultaneously at the boundary between hyperbolic and elliptic transformations, so that the classification in figure 1 also holds for $\tilde{\mathbf{B}}$, providing that one interchanges $0 \leftrightarrow \infty$. If $N=1$, both signatures $\sigma(\mathbf{B})= \pm 2$ and $\sigma(\tilde{\mathbf{B}})= \pm 2$ for an elliptic transformation, whereas $\sigma=0$ for both parametrizations in the case of a hyperbolic transformation.

\section{Metaplectic operators in the Weyl and the chord representations}

For the present purpose, the most appropriate way to consider the Weyl representation of an operator $\hat{A}$, i.e. its centre symbol or Weyl symbol, is

$$
A(\mathbf{x})=2^{N} \operatorname{tr}\left(\hat{R}_{\mathbf{x}} \hat{A}\right),
$$

where $\hat{R}_{\mathbf{x}}$ is the quantum operator corresponding to a reflection through the phase space point $\mathbf{x}$ [2, 26, 27. Within a translation, this is just the symplectic transformation with $\mathbf{M}=-\mathbf{I}, \tilde{\mathbf{B}}=\mathbf{0}$, though its main Cayley matrix $\mathbf{B}$ has a singular limit. It is important to note that such an operator is only defined within an overall phase, but in all standard definitions, one imposes that $\left(\hat{R}_{\mathbf{x}}\right)^{2}=\widehat{I}$.

The metaplectic operator, corresponding to the symplectic transformation $\boldsymbol{x}^{+}=$ $\mathbf{M} \boldsymbol{x}^{-}$and Cayley matrix $\mathbf{B}$, has as Weyl symbol the Weyl propagator,

$U_{\mathbf{M}}(\mathbf{x}) \equiv \pm \frac{2^{N}}{[\operatorname{det}(\mathbf{I}+\mathbf{M})]^{1 / 2}} \exp \left(\frac{\mathrm{i}}{\hbar} \mathbf{x} \cdot \mathbf{B} \mathbf{x}\right)= \pm[\operatorname{det}(\mathbf{I}+\mathbf{J B})]^{1 / 2} \exp \left(\frac{\mathrm{i}}{\hbar} \mathbf{x} \cdot \mathbf{B} \mathbf{x}\right)$,

which was derived in [2] only within a phase. Thus, it should be noted that both amplitudes have here been specified by the square root of a determinant, rather than that of its modulus. There will then be a factor of $i$, if the determinant is negative. In other words, the duplicity of the metaplectic sheet coincides with that of the Riemann sheet for the square root, as shall be verified further on. The $( \pm)$ signs which distinguish the pair of sheets are kept as a reminder that either choice can 
result for a metaplectic operator labelled by the same $\mathbf{M}$, depending on its previous evolution. \&

One should keep in mind that for general motions the only difference between this tangent propagator and the approximate SC Weyl propagator, corresponding to a nonlinear transformation, is that the action of the former is obtained as the homogeneous second order approximation to the full action $S(\mathbf{x})$. (It follows that the amplitude of the tangent propagator is constant.) In other words, (2.4) may either be interpreted as the local phase of the SC propagator, or the exact phase of the tangent propagator. Thus, in the neighbourhood of the origin, which corresponds to the midpoint of a trajectory for the full nonlinear motion, one can identify the overall sign of both propagators.

The alternative forms for the determinantal amplitudes are immediately interpreted with the aid of the transformations between midpoints and endpoints:

$$
\mathbf{x}=\frac{1}{2}(\mathbf{I}+\mathbf{M}) \boldsymbol{x}^{-} \text {or } \boldsymbol{x}^{-}=(\mathbf{I}+\mathbf{J B}) \mathbf{x}
$$

whereas

$$
\mathbf{x}=\frac{1}{2}\left(\mathbf{I}+\mathbf{M}^{-1}\right) \boldsymbol{x}^{+} \text {or } \boldsymbol{x}^{+}=(\mathbf{I}-\mathbf{J B}) \mathbf{x}
$$

Thus, the Jacobians for these transformations are

$$
\operatorname{det}(\mathbf{I} \pm \mathbf{J B})=2^{N} \operatorname{det}(\mathbf{I}+\mathbf{M})^{-1}=2^{N} \operatorname{det}\left(\mathbf{I}+\mathbf{M}^{-1}\right)^{-1},
$$

which also allows for the expression of the amplitude in terms of $\operatorname{det}(\mathbf{I}-\mathbf{J B})$, as in 2]. In the present text, the choice of sign within the determinant in the LHS of (3.5) is never altered in order to avoid any confusion with the issue of determining the overall Maslov phases.

Any continuous family of symplectic transformations corresponds to a continuous family of metaplectic operators. If these are represented by their Weyl symbol, then the latter must also be continuous, except at the caustics, where $\operatorname{det}(\mathbf{B}) \rightarrow \infty$. Recalling that the Weyl representation of the operator $\widehat{I}$ is just the constant, $I(\mathbf{x})=1$, it follows that all the metaplectic operators that are continuously connected to $\widehat{I}$ are correctly described by the $(+)$ sign in (3.2). It will be verified further along that the pair of metaplectic sheets is correctly accounted for, including caustic traversals, by the square roots of these determinants, without taking their modulus.

The Weyl propagator for the harmonic oscillator $(N=1)$, driven by the Hamiltonian $H(x)=(\omega / 2)\left(p^{2}+q^{2}\right)$, provides an iluminating example. After a time $t$, the Cayley matrix for this typical elliptic propagator will be $\mathbf{B}(t)=-\tan (\omega t / 2) \mathbf{I}$, so that the full Weyl propagator is

$$
U_{t}(\mathbf{x})=\frac{1}{\cos (\omega t / 2)} \exp \left[-\frac{\mathrm{i}}{\hbar} \tan (\omega t / 2)\left(p^{2}+q^{2}\right)\right] .
$$

One should note the simple form taken by the square root in the amplitude of (3.2). The Maslov theory in the next section will confirm that the change of phase across the caustic is just that shown in (3.6), in which the sign ambiguity is fully accounted for by adding an even or odd factor of $2 \pi$ to the cosine phase. So there is a minus sign as the boundary $|\omega t|=\pi$ is crossed, coinciding with the sign of the cosine.

$\S$ Other representations of the metaplectic operators, such as the usual position propagator, are also exact in their SC form. One can identify $\mathbf{M} \mapsto \widehat{U}_{\mathbf{M}}$ in all cases, but the special appeal of the present phase space representations is that they are specified by invariants of the full corresponding symplectic matrix, M. 
This signifies a change of metaplectic sheet. At $\omega t=2 \pi$, one obtains $-\widehat{I}$, that is, $U(\mathbf{x})=-1$ and it is only after a second rotation in phase space that the identity operator is regained. In the absence of the history of the evolution, the $( \pm)$ sign is undetermined, but our general concern are the particular phase jumps for particular caustic transitions. Products of metaplectic operators for the harmonic oscillator are discussed in the Appendix.

In contrast, for the inverted harmonic oscillator, $H(x)=(\lambda / 2)\left(p^{2}-q^{2}\right)$, the Weyl propagator is

$$
U_{t}(\mathbf{x})=\frac{1}{\cosh (\lambda t / 2)} \exp \left[-\frac{\mathrm{i}}{\hbar} \tanh (\lambda t / 2)\left(p^{2}-q^{2}\right)\right] .
$$

In the case of such a quadratic hyperbolic Hamiltonian, there is no caustic singularity. Above, we have portrayed the propagators continuously connected to $\widehat{I}$, whereas those in the neighbourhood of $-\widehat{I}$ would all have a negative sign. The hyperbolic transformations with reflection (see figure 1) cannot be reached continuously through the action of a hyperbolic Hamiltonian. Even so, to complete the types of symplectic transformation portrayed in figure 1, one may add the continuous family of operators (for $t$ positive or negative):

$$
U_{t}(\mathbf{x})= \pm \frac{\mathrm{i}}{\sinh (\lambda t / 2)} \exp \left[-\frac{\mathrm{i}}{\hbar} \operatorname{coth}(\lambda t / 2)\left(p^{2}-q^{2}\right)\right] .
$$

However, it should be noted that the corresponding hyperbolic transformations with reflection are continuously obtained from the reflection, for which $\mathbf{M}=-\mathbf{I}$ and $\tilde{\mathbf{B}}=0$, while they cannot be reached directly from $\mathbf{I}$, without traversing a caustic. For this reason the phase presented in (3.8) anticipates the theory in the following sections. One should note that typical metaplectic operators for elliptic and hyperbolic transformations are simply obtained from these examples, owing to the invariance of the Weyl representation with respect to symplectic transformations.

The Cayley matrix for the harmonic oscillator becomes singular for $\omega t=\pi$, when the transformation is just a reflection. The correct corresponding Weyl propagator is a Dirac delta-function, which is indeed singular. In general, a continuous family of metaplectic operators has a singular Weyl representation as $\operatorname{det}(\mathbf{I}+\mathbf{M}) \rightarrow 0$. If $N=1$, this is equivalent to $\operatorname{tr}(\mathbf{M})+2 \rightarrow 0$.

The chord representation of an operator $\hat{A}$, i.e. its chord symbol, is

$$
\tilde{A}(\boldsymbol{\xi})=\operatorname{tr}\left(\hat{T}_{-\boldsymbol{\xi}} \hat{A}\right),
$$

where $\hat{T}_{\boldsymbol{\xi}}$ is a Heisenberg operator, that is, the unitary operator corresponding to a phase space translation by the vector $\boldsymbol{\xi}$. (It is referred to in various ways throughout the literature, but here we follow the notation in 2.) This is the conjugate representation to the Weyl representation, so that the chord propagator corresponding to the same metaplectic operator and, hence, the same classical symplectic matrix, M, can be evaluated through the symplectic Fourier transforms (see e.g. 2]),

$$
\begin{aligned}
& \tilde{U}(\boldsymbol{\xi})=\frac{1}{(2 \pi \hbar)^{N}} \int \mathrm{d} \mathbf{x} \exp \left(\frac{\mathrm{i}}{\hbar} \mathbf{x} \wedge \boldsymbol{\xi}\right) U(\mathbf{x}), \\
& U(\mathbf{x})=\frac{1}{(2 \pi \hbar)^{N}} \int \mathrm{d} \boldsymbol{\xi} \exp \left(\frac{\mathrm{i}}{\hbar} \boldsymbol{\xi} \wedge \mathbf{x}\right) \tilde{U}(\boldsymbol{\xi}),
\end{aligned}
$$

where we recall that the wedge product $\mathbf{x} \wedge \boldsymbol{\xi} \equiv \boldsymbol{\xi} \cdot \mathbf{J x}$. 
To obtain the metaplectic chord propagator, one takes the Fourier transform of the complex Gaussian form of the Weyl propagator (3.2),

$$
\tilde{U}_{\mathbf{M}}(\boldsymbol{\xi})= \pm[\operatorname{det}(\mathbf{I}+\mathbf{J B})]^{1 / 2} \int \frac{\mathrm{d} \mathbf{x}}{(2 \pi \hbar)^{N}} \exp \left[\frac{\mathrm{i}}{\hbar}(\mathbf{x} \cdot \mathbf{B x}-\mathbf{x} \cdot \mathbf{J} \boldsymbol{\xi})\right]
$$

and uses (2.14) so as to express the chord propagator in terms of the alternative Cayley matrix, $\tilde{\mathbf{B}}$, defined by (2.13). Then the amplitude of the chord propagator results from the equality

$$
\operatorname{det}(\mathbf{I}+\mathbf{J} \tilde{\mathbf{B}})=\operatorname{det}(\mathbf{I}+\mathbf{J B}) \operatorname{det}(\mathbf{B})^{-1} .
$$

If the number of negative eigenvalues of the $2 N$-dimensional matrix $\mathbf{B}$ is $N_{-}$, then its signature is $\sigma(\mathbf{B})=2\left(N-N_{-}\right)$, so that the Gaussian integral gains the phase $\pi\left(N-N_{-}\right) / 2$. On the other hand, one can also express $\arg [\operatorname{det}(\mathbf{B})]=\pi\left[N_{-}(\bmod 2)\right]$, so that the new phase can be incorporated into $\operatorname{det}(\mathbf{I}+\mathbf{J} \tilde{\mathbf{B}})^{1 / 2}$, within a phase $\nu \pi$, for some integer $\nu$. This leads to

$$
\begin{aligned}
\tilde{U}_{\mathbf{M}}(\boldsymbol{\xi}) & = \pm(\mathrm{i})^{N}[\operatorname{det}(\mathbf{I}+\mathbf{J} \tilde{\mathbf{B}})]^{1 / 2} \exp \left(-\frac{\mathrm{i}}{4 \hbar} \boldsymbol{\xi} \cdot \tilde{\mathbf{B}} \boldsymbol{\xi}\right) \\
& = \pm \frac{(2 \mathrm{i})^{N}}{[\operatorname{det}(\mathbf{I}-\mathbf{M})]^{1 / 2}} \exp \left(-\frac{\mathrm{i}}{4 \hbar} \boldsymbol{\xi} \cdot \tilde{\mathbf{B}} \boldsymbol{\xi}\right),
\end{aligned}
$$

where the overall $( \pm)$ sign need not coincide with the original Weyl representation (i.e. positive near the identity) and all further phases are specified by the square roots of the determinant. One can also interpret both forms of the amplitude in terms of the relation between the endpoint and the chord:

$$
\boldsymbol{\xi}=[\mathbf{M}-\mathbf{I}] \boldsymbol{x}^{-} \text {or } \boldsymbol{x}^{-}=\frac{1}{2}[\mathbf{J} \tilde{\mathbf{B}}-\mathbf{I}] \boldsymbol{\xi} .
$$

In practice, the increment in phase resulting from the Fourier transform,

$$
\Theta=\frac{\pi}{4} \sigma(\mathbf{B})=\frac{\pi}{2}\left(N-N_{-}\right)
$$

is just added onto the overall preexisting phase in the Weyl representation (i.e. zero, in a causticless neighbourhood of the identity), so as to determine the overall phase in (3.14). For example, one may perform directly the symplectic Fourier integral on (3.6) for the harmonic oscillator. The overall sign for the Weyl propagator is $(+)$ for $\omega t<\pi$, so the choice of sign for the chord propagator will correspond to the phase $(\pi / 4) \sigma(\mathbf{B})=-(\pi / 2) \omega t /|\omega t|$, which leads to

$$
\tilde{U}_{t}(\boldsymbol{\xi})=-\frac{\mathrm{i}}{2 \sin (\omega t / 2)} \exp \left[\frac{\mathrm{i}}{4 \hbar} \cot (\omega t / 2)\left(\boldsymbol{\xi}_{p}{ }^{2}+\boldsymbol{\xi}_{q}{ }^{2}\right)\right] .
$$

Likewise, the Fourier transform for the inverted oscillator (3.7) leads to the chord propagator:

$$
\tilde{U}_{t}(\boldsymbol{\xi})=\frac{1}{2 \sinh (\lambda t / 2)} \exp \left[\frac{\mathrm{i}}{4 \hbar} \operatorname{coth}(\lambda t / 2)\left(\boldsymbol{\xi}_{p}{ }^{2}+\boldsymbol{\xi}_{q}{ }^{2}\right)\right] .
$$

whereas the reflected hyperbolic chord propagator, obtained from (3.8), becomes

$$
\tilde{U}_{t}(\boldsymbol{\xi})=-\frac{\mathrm{i}}{2 \cosh (\lambda t / 2)} \exp \left[\frac{\mathrm{i}}{4 \hbar} \tanh (\lambda t / 2)\left(\boldsymbol{\xi}_{p}{ }^{2}+\boldsymbol{\xi}_{q}{ }^{2}\right)\right] .
$$

\| Note that this corrects the sign of the exponent in (6.40) of reference [2]. 
Here, one should remark that both the oscillator, for $\omega t \rightarrow \pi$, and the reflected hyperbolic family for $\lambda \rightarrow 0$ correspond to a reflection about the origin, but this operator does not have the same phase as the operator $\hat{R}_{\mathrm{x}}$ employed in the definition of the Weyl representation. Indeed, the chord symbol of the standard reflection is $R_{\mathbf{x}}(\boldsymbol{\xi})=2^{-N}$, so that one should distinguish the present metaplectic reflection as $\hat{R}_{\mathbf{x}}^{\prime}=\mathrm{i}^{-N} \hat{R}_{\mathbf{x}}$ (i.e. $\left.R_{\mathbf{x}}^{\prime}(\boldsymbol{\xi})=(2 \mathrm{i})^{-N}\right)$, which satisfies $\left(\hat{R}_{\mathbf{x}}^{\prime}\right)^{2}=(-1)^{N} \widehat{I}$. This subtle phase distinction has surely been anticipated in other contexts (e.g. in [28), but it is for manipulations in the Weyl representation that it is of crucial importance.

Returning to the full expression for the metaplectic chord propagator (3.14), one realizes that the limit $\tilde{\mathbf{B}} \rightarrow 0$ must specify the metaplectic reflection, $\pm \hat{R}_{0}^{\prime}$. Indeed, all operators that are continuously connected to such a reflection will preserve the same overall sign and the boundary of this region is only reached as one of the eigenvalues of $\tilde{\mathbf{B}}$ reaches the value +1 . Thus there is no caustic for the chord representation as an eigenvalue of $\tilde{\mathbf{B}}$ goes through zero, even though there may be a switch of type (elliptic $\leftrightarrow$ hyperbolic, if $N=1$ ). In the previous examples, one thus verifies that the transition between the chord propagator for the harmonic oscillator (as $\omega t \rightarrow \pi$ ) and the hyperbolic with reflection (as $\lambda t \rightarrow 0$ ) is smooth. Furthermore, all chord propagators within a causticless neighbourhood of the reflection, $\hat{R}_{0}^{\prime}$, corresponding to the phase $\pi$ of the harmonic oscillator, share the overall phase $-\pi / 2$.

This scenario is just the complement of that previously observed for the Weyl representation, in which all operators that are continuously connected to the identity must share the same overall sign. In other words, the continuous group of metaplectic operators is represented with a cut in the Weyl representation that includes the reflection, while its cut in the chord representation includes the identity. Each representation is smooth along the other's singularity. This is the basis of the Maslov method of phase determination in the following section.

The alternative approach to determine the Maslov phases is to rely on products of metaplectic operators. At the passage of a one-parameter family of tangent operators through the singularity of its Weyl representation, an operator, characterized by large $|\operatorname{det}(\mathbf{B})|$ or small $|\operatorname{det}(\tilde{\mathbf{B}})|$, is multiplied by an operator close to the identity, i.e. with small $|\operatorname{det}(\mathbf{B})|$. Thus, the determination of the final overall sign should be included in the problem of establishing the overall phase for the product of any pair of metaplectic operators. This is obtained from the product rule in the Weyl representation for arbitrary pairs of operators [2], $\widehat{A}=\widehat{A}_{2} \widehat{A}_{1}$ :

$\left[A_{2} A_{1}\right](\mathbf{x})=\int \frac{\mathrm{d} \mathbf{x}_{2} \mathrm{~d} \mathbf{x}_{1}}{(\pi \hbar)^{2 N}} A_{2}\left(\mathbf{x}_{2}\right) A_{1}\left(\mathbf{x}_{1}\right) \exp \left[\frac{2 \mathrm{i}}{\hbar}\left(\mathbf{x}_{2}-\mathbf{x}\right) \cdot \mathbf{J}\left(\mathbf{x}_{1}-\mathbf{x}\right)\right]$.

In the case of metaplectic operators, each Weyl propagator is a complex Gaussian, so that (3.20) is a $4 N$-dimensional Gaussian integral. This is reduced to a $2 N$ dimensional integral in the limiting case where either $\mathbf{B}=0$ or $\tilde{\mathbf{B}}=0$, for one of the factors (i.e. it is either a translation or a reflection). These special cases are considered in section 5 .

\section{Generalized Maslov method for the overall sign of the propagator}

Continuity of the family of tangent operators (corresponding to the neighbourhood of a trajectory with a given initial value) implies the continuity of the family of tangent Weyl propagators in a causticless neighbourhood of the identity operator. Hence, the overall sign of the SC approximation to the full Weyl propagator must be preserved in 
this region, regardless of the type of symplectic transformation (hyperbolic or elliptic, if $N=1$ ): A transition between these two types within this neighbourhood, at a tangent propagator characterized by (2.9), does not alter the overall sign.

This scenario is in marked contrast to the chord propagator in this same region, which has its caustic exactly for those transformations characterized by (2.9). The change of phase across this boundary is then evaluated by just the procedure followed in the previous section, that is, by performing the Fourier transform from the Weyl propagator. This is a generalization of the Maslov method [7] that has already been tacitly employed to obtain the change of sign for the harmonic oscillator (3.17) at $t=0$.

The same method can now be employed to explore the possible change of phase within the Weyl representation across its caustic, characterized by (2.15). What then is the result of reversing the Fourier transformation, so as to return to the tangent Weyl propagator from the chord propagator? There is certainly no change if no caustic has been crossed, since this procedure may be continuously connected to the simple task of performing and then reversing the same Fourier transformation. In terms of Gaussian integrals, this general conclusion follows from the important result in section 2, that both Cayley matrices for a given transformation $\mathbf{M}$, i.e. $\mathbf{B}$ and $\tilde{\mathbf{B}}$, have the same signature.

Let us now consider a one-parameter family of tangent operators, which evolves from an original operator, characterized by $(\mathbf{M}, \mathbf{B}, \tilde{\mathbf{B}})$, such that $\sigma(\mathbf{B})=\sigma(\tilde{\mathbf{B}})=$ $2\left(N-N_{-}\right)$, to a new operator, characterized by $\left(\mathbf{M}^{\prime}, \mathbf{B}^{\prime}, \tilde{\mathbf{B}}^{\prime}\right)$, with $\sigma\left(\mathbf{B}^{\prime}\right)=\sigma\left(\tilde{\mathbf{B}}^{\prime}\right)=$ $2\left(N-N_{-}^{\prime}\right)$. If a single Weyl caustic is crossed, the continuity of this operator evolution is still maintained within the chord representation, so that there is no change of the overall phase of the chord propagator. The increment of the overall phase of the Weyl propagator will then just depend on the pair of Fourier integrals:

$$
\Theta=\frac{\pi}{4}\left[\sigma\left(\mathbf{B}^{\prime}\right)-\sigma(\mathbf{B})\right]=\frac{\pi}{2}\left(N_{-}-N_{-}^{\prime}\right) .
$$

There is no phase increment if $N_{-}^{\prime}=N_{-}$. Alternatively, $\Theta$ need not equal $\nu \pi$ (with integer $\nu$ ), if the evolution has changed the type of the transformation, being that the square roots in the amplitudes may be imaginary. This is just the case, if $\mathbf{M}$ denoted an elliptic transformation (harmonic oscillator (3.6)), whereas $\mathbf{M}^{\prime}$ becomes a reflected hyperbolic transformation (3.8), though both transformations are smoothly connected in the chord representation.

In general, the sign of the determinant in the final Weyl propagator (3.2) already picks up the signatures for the pair of Fourier transformations, so as to specify the phase of the Weyl propagator within $\pm \pi$. That is, using (3.13) and its reverse, as well as continuity of the chord propagator, one obtains

$$
\begin{aligned}
\arg \left[\operatorname{det}\left(\mathbf{I}+\mathbf{J B}^{\prime}\right)\right] & =\arg \left[\operatorname{det}\left(\mathbf{I}+\mathbf{J} \tilde{\mathbf{B}}^{\prime}\right)\right]-\arg \left[\operatorname{det}\left(\tilde{\mathbf{B}}^{\prime}\right)\right] \\
& =\arg [\operatorname{det}(\mathbf{I}+\mathbf{J B})]-\arg [\operatorname{det}(\mathbf{B})]+\arg \left[\operatorname{det}\left(\mathbf{B}^{\prime}\right)\right],
\end{aligned}
$$

so that the phase increment due to the determinant is just

$$
\arg \left[\operatorname{det}\left(\mathbf{B}^{\prime}\right)\right]-\arg [\operatorname{det}(\mathbf{B})]=\frac{\pi}{2}\left[\left(N_{-}^{\prime}-N_{-}\right)(\bmod 2)\right] .
$$

I In the double phase space scenario for semiclassical approximations, the evolution operator corresponds to a Lagrangian surface expressed in terms of the conjugate centre or chord coordinates [21]. Thus, one obtains complete equivalence to switching between positions and momenta in ordinary phase space.

+ Notice that the exponents of the Weyl propagator and the chord propagator have different signs. 
Comparing with (4.1), one verifies that full knowledge of the signatures $\sigma(\mathbf{B})$ and $\sigma\left(\mathbf{B}^{\prime}\right)$ only distinguishes the final $( \pm)$ sign. In this way, the postulation of the Weyl and the chord representations of metaplectic propagators in terms of determinants without moduli in (3.2) and (3.14) is justified: The determinants automatically account for the correct phase changes across caustics, within an overall sign, which is then specified by (4.1).

Finally, one should note that the overall phase increment for crossing a caustic in the conjugate chord representation, for a continuous evolution that crosses no Weyl caustic, is obtained in perfect symmetry to the above results.

\section{Product with a translation or a reflection}

Operators that correspond to translations or reflections through a point in phase space are the building blocks of, respectively, the chord and the Weyl representation 2, 26, 27. Just as the metaplectic operators, they can also be exactly rendered by semiclassical formulae. Furthermore, the combination of translations and reflections form the quantum affine group, which, together with the metaplectic group, integrate the inhomogeneous metaplectic group [17, corresponding to the (classical) inhomogeneous symplectic group. The generating functions for these transformations add a linear term to the quadratic generating functions of the (homogeneous) symplectic transformations that we have so far considered [2].

Let us first consider the product of a metaplectic operator $\widehat{U}_{1}$, represented by $U_{1}(\mathbf{x})$ according to (3.2) with the unitary (Heisenberg) translation operator,

$$
\hat{T}_{\boldsymbol{\xi}}=\exp \left(\frac{\mathrm{i}}{\hbar} \boldsymbol{\xi} \wedge \hat{\boldsymbol{x}}\right) \text {, so that } T_{\boldsymbol{\xi}}(\mathbf{x})=\exp \left(\frac{\mathrm{i}}{\hbar} \boldsymbol{\xi} \wedge \mathbf{x}\right) \text {, }
$$

which includes $T_{0}(\mathbf{x})=I(\mathbf{x})=1$. Assuming that $\widehat{U}_{1}$ is in the neighbourhood of $\widehat{I}$ for which the overall sign is positive, then, according to (3.20), the product transformation is given by

$$
U_{\boldsymbol{\xi}}(\mathbf{x})= \pm\left[\operatorname{det}\left(\mathbf{I}+\mathbf{J B}_{1}\right)\right]^{1 / 2} \int \frac{\mathrm{d} \mathbf{x}_{2} \mathrm{~d} \mathbf{x}_{1}}{(\pi \hbar)^{2 N}} \exp \left(\frac{\mathrm{i}}{\hbar} \Phi\right)
$$

where

$$
\Phi=\mathbf{x}_{1} \cdot \mathbf{B}_{1} \mathbf{x}_{1}+\mathbf{x}_{2} \cdot \mathbf{J} \boldsymbol{\xi}+2 \mathbf{x}_{2} \cdot \mathbf{J} \mathbf{x}_{1}-2 \mathbf{x}_{2} \cdot \mathbf{J} \mathbf{x}-2 \mathbf{x} \cdot \mathbf{J} \mathbf{x}_{1} .
$$

The integral over $\mathbf{x}_{2}$ is just a Dirac $\delta$-function,

$$
\begin{aligned}
U_{\boldsymbol{\xi}}(\mathbf{x})= \pm & {\left[\operatorname{det}\left(\mathbf{I}+\mathbf{J B}_{1}\right)\right]^{1 / 2} \int \frac{\mathrm{d} \mathbf{x}_{1}}{(\pi \hbar)^{2 N}} } \\
& \times(2 \pi \hbar)^{2 N} \delta\left(2 \mathbf{x}_{1}-2 \mathbf{x}+\boldsymbol{\xi}\right) \exp \left[\frac{\mathrm{i}}{\hbar}\left(\mathbf{x}_{1} \cdot \mathbf{B}_{1} \mathbf{x}_{1}-2 \mathbf{x} \cdot \mathbf{J} \mathbf{x}_{1}\right)\right]
\end{aligned}
$$

so that the result is the inhomogeneous metaplectic transformation,

$U_{\boldsymbol{\xi}}(\mathbf{x})= \pm\left[\operatorname{det}\left(\mathbf{I}+\mathbf{J B}_{1}\right)\right]^{1 / 2} \exp \left[\frac{\mathrm{i}}{\hbar}\left((\mathbf{x}-\boldsymbol{\xi} / 2) \cdot \mathbf{B}_{1}(\mathbf{x}-\boldsymbol{\xi} / 2)+\mathbf{x} \cdot \mathbf{J} \boldsymbol{\xi}\right)\right]$,

that corresponds to the inhomogeneous symplectic transformation generated by the centre generating function,

$$
S_{\boldsymbol{\xi}}(\mathbf{x})=(\mathbf{x}-\boldsymbol{\xi} / 2) \cdot \mathbf{B}_{1}(\mathbf{x}-\boldsymbol{\xi} / 2)+\mathbf{x} \cdot \mathbf{J} \boldsymbol{\xi}
$$


through equations (2.1), while holding the translation chord, $\boldsymbol{\xi}$, as a fixed parameter. One notes that no new phase appears in the integral over the $\delta$-function, that is, the overall sign of $U_{1}(\mathbf{x})$ is preserved. In the limit, $\boldsymbol{\xi} \rightarrow 0$, i.e. for the product with the identity operator, it is verified that $\left[I \cdot U_{1}\right](\mathbf{x})=U_{1}(\mathbf{x})$.

The product of a metaplectic operator with the reflection, $\widehat{R}_{\boldsymbol{y}}$, through the phase space point $\boldsymbol{y}$ also simplifies, because

$$
R_{\boldsymbol{y}}(\mathbf{x})=(\pi \hbar)^{N} \delta(\mathbf{x}-\boldsymbol{y})
$$

in the Weyl representation [2]. Inserting this into (3.20) then leads to

$$
\begin{aligned}
U_{\boldsymbol{y}}(\mathbf{x})= \pm[\operatorname{det}(\mathbf{I} & \left.\left.+\mathbf{J B}_{1}\right)\right]^{1 / 2} \exp \left(-\frac{2 \mathrm{i}}{\hbar} \boldsymbol{y} \cdot \mathbf{J} \mathbf{x}\right) \int \frac{\mathrm{d} \mathbf{x}_{1}}{(\pi \hbar)^{N}} \\
& \times \exp \left[\frac{\mathrm{i}}{\hbar}\left(\mathbf{x}_{1} \cdot \mathbf{B}_{1} \mathbf{x}_{1}-2 \mathbf{x}_{1} \cdot \mathbf{J}(\boldsymbol{y}-\mathbf{x})\right] .\right.
\end{aligned}
$$

The integral has the same form as the Fourier transform of a complex Gaussian in the $2 N$-dimensional phase space (3.12) which led to the chord propagator in section 3 . So this again leads to a metaplectic operator with its Weyl symbol:

$$
U_{\boldsymbol{y}}(\mathbf{x})= \pm \mathrm{i}^{N}\left[\operatorname{det}\left(\mathbf{I}+\mathbf{J} \tilde{\mathbf{B}}_{1}\right)\right]^{1 / 2} \exp \left(-\frac{\mathrm{i}}{\hbar} S_{\boldsymbol{y}}(\mathbf{x})\right),
$$

where

$$
S_{\boldsymbol{y}}(\mathbf{x})=(\mathbf{x}-\boldsymbol{y}) \cdot \tilde{\mathbf{B}}_{1}(\mathbf{x}-\boldsymbol{y})+2 \boldsymbol{y} \cdot \mathbf{J} \mathbf{x}
$$

is the centre generating function for the corresponding inhomogeneous symplectic transformation specified by (2.1). In the case of a reflection through the origin (i.e. a parity operator), $\boldsymbol{y}=0$ and (5.9) will be just the homogeneous metaplectic operator specified by $-\tilde{\mathbf{B}}_{1}$, i.e. the product propagator adopts the Cayley matrix of the original chord propagator.

The factor $\mathrm{i}^{N}$ may seem strange, since it does not fit in with the phases in (3.2). If one chooses $U_{1}(\mathbf{x})$ for a harmonic osillator, the product may be matched to just a further rotation in phase space by $\pi / 2$, so that presumably (5.9) should satisfy (3.6). Thus, one should recall the conclusion in section 3 that the appropriate reflection included in the metaplectic group is not $\hat{R}_{\mathbf{x}}$, but the operator $\hat{R}_{\mathbf{x}}^{\prime}=\mathrm{i}^{-N} \hat{R}_{\mathbf{x}}$. For the product of this metaplectic reflection with a general metaplectic operator, the factor of $\mathrm{i}^{N}$ is indeed cancelled in the corresponding expression to (5.9). In applications where the product arises because evolution acts on an operator specified by its Weyl symbol, this extra factor must be included, because the reflection operator implied will then indeed be $\hat{R}_{\mathbf{x}}$, rather than $\hat{R}_{\mathbf{x}}^{\prime}$.

In practice, the phase increment due to the product by $\hat{R}_{\mathbf{x}}$ is just

$$
\Theta=\frac{\pi}{4} \sigma\left(\mathbf{B}_{1}\right)=\frac{\pi}{2}\left(N-N_{1-}\right),
$$

where $N_{1-}$ is the number of negative eigenvalues of $\mathbf{B}_{1}$. This determines the $( \pm)$ sign and it is exactly the same phase increment as previously obtained in passing from the Weyl propagator to the chord propagator in section 3 ,

For the sake of brevity, we note that in the chord representation products of translations or reflections with metaplectic operators are just Fourier transforms of (5.5) and (5.9). Basically, the corresponding Cayley matrices for factors and their products are given by (2.14). Here again, one must carefully choose between $\hat{R}_{\mathrm{x}}$ and $\hat{R}_{\mathbf{x}}^{\prime}$, depending on the context: their chord symbols are Dirac $\delta$-functions with different phase factors. 


\section{Products of metaplectic transformations}

We now consider general products of pairs of arbitrary metaplectic transformations, $\widehat{U}=\widehat{U}_{2} \widehat{U}_{1}$, corresponding to symplectic transformations, such that $\mathbf{M}=\mathbf{M}_{2} \mathbf{M}_{1}$, parametrized by Cayley matrices, $\mathbf{B}_{1}$ and $\mathbf{B}_{2}$, according to (2.5), so that both factors take on the form (3.2). For simplicity, we shall assume that both $\widehat{U}_{1}$ and $\widehat{U}_{2}$ have overall positive signs. Inserting these ingredients into the product rule (3.20) then leads to

$U(\mathbf{x})=\left[\operatorname{det}\left(\mathbf{I}+\mathbf{J B}_{1}\right)\right]^{1 / 2}\left[\operatorname{det}\left(\mathbf{I}+\mathbf{J B}_{2}\right)\right]^{1 / 2} \int \frac{\mathrm{d} \mathbf{x}_{2} \mathrm{~d} \mathbf{x}_{1}}{(\pi \hbar)^{2 N}} \exp \left(\frac{\mathrm{i}}{\hbar} \Phi\left(\mathbf{x}, \mathbf{x}_{1}, \mathbf{x}_{2}\right)\right)$,

where

$\Phi\left(\mathbf{x}, \mathbf{x}_{1}, \mathbf{x}_{2}\right)=\mathbf{x}_{1} \cdot \mathbf{B}_{1} \mathbf{x}_{1}+\mathbf{x}_{2} \cdot \mathbf{B}_{2} \mathbf{x}_{2}+2 \mathbf{x}_{2} \cdot \mathbf{J x}_{1}-2 \mathbf{x}_{2} \cdot \mathbf{J} \mathbf{x}-2 \mathbf{x} \cdot \mathbf{J} \mathbf{x}_{1}$

is a homogeneous quadratic form in its $6 N$ variables, or an inhomogeneous quadratic form in the $4 N$ variables $\left(\mathbf{x}_{1}, \mathbf{x}_{2}\right)$ if we fix the centre $\mathbf{x}$ where the full propagator is evaluated. It is shown in 2 that, upon obtaining $\mathbf{x}_{1}(\mathbf{x})$ and $\mathbf{x}_{2}(\mathbf{x})$ from the conditions, $\partial \Phi / \partial \mathbf{x}_{1}=0$ and $\partial \Phi / \partial \mathbf{x}_{2}=0$, the resulting quadratic form in $\mathbf{x}$ is just the centre generating function for the product transformation, that is, $\Phi\left(\mathbf{x}, \mathbf{x}_{1}(\mathbf{x}), \mathbf{x}_{2}(\mathbf{x})\right)=$ $S(\mathbf{x})=\mathbf{x} \cdot \mathbf{B x}$, corresponding to $\mathbf{M}=\mathbf{M}_{2} \mathbf{M}_{1}$. Adopting this stationary point of $\left(\mathbf{x}_{1}, \mathbf{x}_{2}\right)$ as the origin then leads to

$$
\begin{aligned}
& U(\mathbf{x})=\left[\operatorname{det}\left(\mathbf{I}+\mathbf{J B}_{1}\right)\right]^{1 / 2}[\operatorname{det}\left.\left(\mathbf{I}+\mathbf{J B}_{2}\right)\right]^{1 / 2} \exp \left(\frac{\mathrm{i}}{\hbar} \mathbf{x} \cdot \mathbf{B} \mathbf{x}\right) \\
& \times \int \frac{\mathrm{d} \mathbf{x}_{2} \mathrm{~d} \mathbf{x}_{1}}{(\pi \hbar)^{2 N}} \exp \left(\frac{\mathrm{i}}{\hbar} \Phi\left(0, \mathbf{x}_{1}, \mathbf{x}_{2}\right)\right),
\end{aligned}
$$

so that our task is to determine the amplitude and phase for the $4 N$-dimensional complex Gaussian integral in (6.3).

One must evaluate the determinant of the $4 N$-dimensional matrix [B $-\mathbf{J}]$, where one defines the symmetric block matrices:

$$
\mathbf{B} \equiv\left(\begin{array}{cc}
\mathbf{B}_{1} & 0 \\
0 & \mathbf{B}_{2}
\end{array}\right) \text { and } \mathbf{J} \equiv\left(\begin{array}{cc}
0 & \mathbf{J} \\
-\mathbf{J} & 0
\end{array}\right) \text {. }
$$

The phase increment is then

$$
\Theta=\frac{\pi}{4} \sigma(\mathbf{B}-\mathbf{J})=\pi\left(N-\frac{\mathrm{N}_{-}}{2}\right),
$$

where $\mathrm{N}_{-}$is the number of negative eigenvalues. In the limit $\mathbf{B} \rightarrow 0$, one is left with $-\mathbf{J}$, which has $2 N$ eigenvalues +1 and $2 N$ eigenvalues -1 , so that $\operatorname{det}(\mathbf{B}-\mathbf{J})=1$ and $\sigma(\mathbf{B}-\mathbf{J})=0$. This confirms a basic assumption for previous results in this paper: There is no overall change of sign for the product transformation, as long as the matrix $\mathbf{B}$ can be treated as a small perturbation of $\mathbf{J}$. Indeed, for a continuous one-parameter family of matrices $\mathbf{B}$, it is only at $\operatorname{det}(\mathbf{B}-\mathbf{J})=0$, as an eigenvalue vanishes, that there may occur an overall change of phase, unless either of the matrices $\mathbf{B}_{j}$ becomes singular.

Just as in the Fourier transform between the Weyl and the chord propagators in section 3, the phase of the determinant of the relevant quadratic form already captures part of the information supplied by its signature, i.e. $\arg [\operatorname{det}(\mathbf{B}-\mathbf{J})]=\pi\left[\mathrm{N}_{-}(\bmod 2)\right]$. The block form of the $4 N$-dimensional determinant allows for the simplification:

$$
\operatorname{det}(\mathbf{B}-\mathbf{J})=(-1)^{2 N} \operatorname{det}\left(\begin{array}{cc}
-\mathbf{J} & \mathbf{B}_{1} \\
\mathbf{B}_{2} & \mathbf{J}
\end{array}\right)=\boldsymbol{\Delta}
$$


where we define,

$$
\boldsymbol{\Delta} \equiv \operatorname{det}\left(\mathbf{I}+\mathbf{J B}_{2} \mathbf{J B}_{1}\right)=\operatorname{det}\left(\mathbf{I}+\mathbf{J B}_{1} \mathbf{J B}_{2}\right) .
$$

Further understanding of the $2 N$-dimensional matrix with this determinant is gained by drawing back to the interpretation of the amplitude of the Weyl propagators in terms of the matrix (3.3) between the midpoint and the endpoint of the classical trajectory. For the product transformation with symplectic matrices $\mathbf{M}=\mathbf{M}_{2} \mathbf{M}_{1}$, we then have

$$
\frac{1}{2}(\mathbf{I}+\mathbf{M})=\left(\mathbf{I}+\mathbf{J B}_{2}\right)^{-1}\left(\mathbf{I}+\mathbf{J B}_{2} \mathbf{J B}_{1}\right)\left(\mathbf{I}+\mathbf{J B}_{1}\right)^{-1} .
$$

The alternative form of (6.7) arises similarly from the inverse product transformation to (6.8), i.e. $\mathbf{M}^{-1}=\mathbf{M}_{1}{ }^{-1} \mathbf{M}_{2}{ }^{-1}$ is related to

$$
\frac{1}{2}\left(\mathbf{I}+\mathbf{M}^{-1}\right)=\left(\mathbf{I}-\mathbf{J B}_{1}\right)^{-1}\left(\mathbf{I}+\mathbf{J B}_{1} \mathbf{J B}_{2}\right)\left(\mathbf{I}-\mathbf{J B}_{2}\right)^{-1} .
$$

Hence, the amplitude of the Weyl propagator for the product operator follows from

$$
2^{2 N} \operatorname{det}(\mathbf{I}+\mathbf{M})^{-1}=\operatorname{det}\left(\mathbf{I}+\mathbf{J B}_{2}\right) \operatorname{det}\left(\mathbf{I}+\mathbf{J B}_{1}\right) \boldsymbol{\Delta}^{-1} .
$$

The conclusion is that the product of a pair of metaplectic operators in the Weyl representation (3.2) results in a Weyl propagator of exactly the same form, but expressed in terms of the Cayley matrix for the combined symplectic transformation. The indeterminacy of the overall sign in the square root of the determinantal amplitude can only be lifted by evaluating the phase $\Theta$ in (6.5). In the case of a continuous oneparameter family of product transformations, i.e. a one-parameter family of double matrices $\mathbf{B}$ (or, in the simplest case, a one-parameter family of matrices $\mathbf{B}_{j}$, with the other matrix held fixed) a possible change of the overall phase of the product only arises if there is a change of sign of $\operatorname{det}(\mathbf{B}-\mathbf{J})=\boldsymbol{\Delta}$. But, according to (6.8), this event entails a change of sign for the final $\operatorname{det}(\mathbf{I}+\mathbf{M})$, itself. So there is no change of phase unless the combined symplectic transformation crosses a caustic.

It is often the case that $\widehat{U}_{2}$ can be identified with a member of a family $\widehat{U}_{t}$ for $t=t_{2}$, while $\widehat{U}_{t=0}=\widehat{I}$; for instance, the symplectic Hamiltonian flow: $\mathbf{M}=\exp [t \mathbf{J H}]$, where $\mathbf{H}$ is the Hamiltonian matrix. Here, $\widehat{U}_{1}$ is an arbitrary passive metaplectic operator. Then, if $\operatorname{det}\left(\mathbf{M}_{t} \mathbf{M}_{1}-\mathbf{I}\right) \neq 0$ from $t=0$ until $t=t_{2}$, the phase increment for a product can be obtained from the difference of signatures of $2 N$-dimensional matrices (4.1). One must be wary of the change of notation, since the transition of the Cayley matrices in section 4 , which was $\mathbf{B} \mapsto \mathbf{B}^{\prime}$, becomes here $\mathbf{B}_{1} \mapsto \mathbf{B}$.

The advantage of this alternative approach relying on continuity is that it only deals with $2 N$-dimensional matrices. In the case of the chord representation, even the products of metaplectic operators can be considered within a $2 \mathrm{~N}$-dimensional integral, because of its simpler product rule. The deduction of the double sheet structure of metaplectic operators has previously been achieved by Littlejohn 17 within the position representation and for the chord representation (with different notation) by de Gosson [18. Our object here is not merely to show how the group of metaplectic transformations is represented by Weyl symbols, but above all to determine the phase increment in each specific case.

It may happen that $\boldsymbol{\Delta}$ and hence $\operatorname{det}(\mathbf{B}-\mathbf{J})$ has a double root for a family of operator products. An important instance of this occurs if the commutator $\left[\mathbf{J B}_{2}, \mathbf{J B}_{1}\right]=0$. This includes the case where $\mathbf{M}=\left(\mathbf{M}_{1}\right)^{2}$, or where $\mathbf{M}_{1}$ and $\mathbf{M}_{2}$ are generated by the same quadratic Hamiltonian. Then $\mathbf{J B}_{1}$ and $\mathbf{J B}_{2}$ can be simultaneously diagonalized, such that $\lambda_{1}^{(k)}$ and $\lambda_{2}^{(k)}$ are their eigenvalues for the 
common $k$-th eigenvector of both these matrices. Hence, the caustic condition for the product transformation, $\Delta=0$, reduces to $\lambda_{1}^{(k)} \lambda_{2}^{(k)}=-1$. This condition allows for elliptic transformations, but not direct hyperbolic transformations, for which $\left|\lambda_{j}\right|<1$. On the other hand, $-\lambda_{j}^{(k)}$ will also be a pair of simultaneous eigenvalues, so that $\Delta$ has a double root. The change of phase at the caustic is then an integral multiple of $\pi$, which is consistent with the need for a product of elliptic transformations to remain elliptic, i.e. there can be no transition to hyperbolic with reflection.

An example of a single null eigenvalue occurs at the caustic of a family of products of elliptic with hyperbolic transformations:

$$
\begin{aligned}
& \mathbf{B}_{1}=\omega\left(\begin{array}{ll}
1 & 0 \\
0 & 1
\end{array}\right), \mathbf{B}_{2}=\gamma\left(\begin{array}{cc}
1 & 0 \\
0 & -1
\end{array}\right) \\
& \mathbf{I}+\mathbf{J B}_{1} \mathbf{J B}_{2}=\left(\begin{array}{cc}
1+\gamma \omega & 0 \\
0 & 1-\gamma \omega
\end{array}\right) .
\end{aligned}
$$

Thus, defining the one-parameter family of transformations either by the parameter $\gamma$ or by the parameter $\omega$, there will be a single null eigenvalue, i.e. single zero of $\boldsymbol{\Delta}$, at $\gamma \omega=1$ and another one at $\gamma \omega=-1$. The symplectic matrices corresponding to (6.11) are

$\mathbf{M}_{1}=\frac{1}{1+\omega^{2}}\left(\begin{array}{cc}1-\omega^{2} & 2 \omega \\ -2 \omega & 1-\omega^{2}\end{array}\right), \mathbf{M}_{2}=\frac{1}{1-\gamma^{2}}\left(\begin{array}{cc}1+\gamma^{2} & -2 \gamma \\ -2 \gamma & 1+\gamma^{2}\end{array}\right)$.

For the caustic condition $\gamma \omega=1$, one obtains

$$
\mathbf{I}+\mathbf{M}=\frac{4}{\omega^{2}-\gamma^{2}}\left(\begin{array}{cc}
1 & \omega \\
-\gamma & -1
\end{array}\right)
$$

so that $\operatorname{det}(\mathbf{I}+\mathbf{M})=\operatorname{tr}(\mathbf{M})+2=0$, but $\mathbf{M}$ is not a reflection. One can check that $\operatorname{det}(\mathbf{I}+\mathbf{M})$ does change its sign as the product parameter $\gamma \omega$ passes the value 1, which leads to a transition of the type of symplectic transformation for the product.

In all cases, full account must be taken of continuity for the proper family of tangent propagators. Indeed, one or both of the factor families may cross a caustic, without any effect on the overall phase of the product. An important instance is the fidelity, or Loschmidt echo operator treated in [19], such that the product of finite evolutions remains close to the identity operator. Continuity of the product operator constrains the continuity of its Weyl representation, in spite of any possible discontinuities in the phase of the factor propagators. This may considerably simplify practical calculations. The way that the phase jumps cancel for the particular example of a product of harmonic oscillator evolutions is analyzed in the Appendix.

It should be remarked that the SC propagator for Wigner functions may be considered as a special case of a compound propagator resulting from the product of evolution operators. This has the peculiarity that the dominant classical trajectory is precisely on a caustic, so that a higher uniform approximation has been developed, for which the phases were previously analyzed [20, 22]. In contrast, the mixed propagator presented in [21] avoids the caustic, so that its tangent propagator for short times is given by (3.2) with a positive sign. It is shown in [19] that there are important applications where the caustic disappears within an integration, so that one needs only worry about the phase increment, which is the focus of the present study. 
Metaplectic sheets and caustic traversals in the Weyl representation

\section{Conclusions}

Notwithstanding the theoretical interest in the way that the Weyl symbol renders the double sheeted metaplectic group, practical use of this phase space representation requires clear rules for phase increments. Indeed, one should not need to be reminded about the abstract topology of this group so as to evolve quantum operators in the SC approximation. The difficulty lies in the crossing of caustics, either as time changes (while the argument of the Weyl propagator is fixed), or for different arguments of the same Weyl propagator. In both cases, the phase increment coincides with that of the appropriate family of metaplectic operators, each of which corresponds to a definite classical trajectory. Fortunately, one can indeed summarize the conclusions in a nutshell:

(i) Continuity of the family of metaplectic operators, corresponding to the family of tangent maps neighbouring each classical trajectory, guarantees that there will be no SC phase jump, unless a caustic is crossed. This even holds for compound trajectories that are pieced together to form super propagators for Wigner functions, or for SC evaluations of the quantum fidelity. No matter how many caustics may have been traversed by the factor operators, one only needs to worry about phase jumps if the product propagator has crossed a caustic. In a causticless neighbourhood of the origin, the sign for the tangent propagators in (3.2) is positive.

(ii) For each caustic traversal of the Weyl propagator (compound or not), one needs a sample of the classical Cayley matrix for a pair of arbitrary instants before and after this SC singularity is met. This requires, either the quadratic approximation of the centre action (i.e. the centre generating function (2.4) ) or else the relation to the (linearized) symplectic transformation (2.6). Then the phase jump is obtained by the difference in the signature between these Cayley matrices (4.1). By expressing the amplitude of the Weyl propagators in terms of a determinant without a modulus in (3.2), any factor of $i$ is already accounted for beyond the caustic. The remaining ambiguity concerning the final phase of $\pi$ is then resolved by adding the above phase to the phase preceding the caustic traversal. The required information is naturally available in any SC computation.

Examples of these rules for products of propagators corresponding to (exactly metaplectic) harmonic oscillators with different frequencies are discussed in the appendix.

The symmetry between Weyl propagators and their Fourier transform leads to similar phase recipes for chord propagators. They are an equally valuable source of super operators for which one must determine the precise phase. The phase jump upon crossing a caustic is obtained by simply replacing $\mathbf{B}$ and $\mathbf{B}^{\prime}$ in (4.1) by $-\tilde{\mathbf{B}}$ and $-\tilde{\mathbf{B}}^{\prime}$.

\section{Appendix A. Product of two oscillations}

Let us consider the product of evolution operators for a pair of harmonic oscillators $(N=1)$, described by the Weyl propagators (3.6). It should be recalled that these may be considered as tangent propagators for general nonquadratic Hamiltonians, such that the corresponding classical tangent maps are both elliptic. The simplifying assumption here is that both maps can be diagonalized simultaneously. Conveniently one keeps a 
common time, $t$, as a single parameter for the product, while the frequency $\omega_{2}$ may differ from $\omega_{1}$, even with respect to its sign, denoting a different sense of oscillation. The Weyl propagator for the product is then

$$
\begin{aligned}
U_{t}(\mathbf{x})=\frac{\exp \left[-\frac{\mathrm{i}}{\hbar}\right.}{\left.\cos \left(\frac{\omega_{1}+\omega_{2}}{2} t\right) \mathbf{x}^{2}\right]} & \int \frac{\mathrm{d} \mathbf{x}_{2} \mathrm{~d} \mathbf{x}_{1}}{(\pi \hbar)^{2}} \\
& \times \exp \left[\frac{\mathrm{i}}{\hbar}\left(\Omega_{1} \mathbf{x}_{1}{ }^{2}+\Omega_{2} \mathbf{x}_{2}{ }^{2}+2 \mathbf{x}_{2} \cdot \mathbf{J} \mathbf{x}_{1}\right)\right] .
\end{aligned}
$$

where

$$
\Omega_{j}=-\tan \left(\frac{\omega_{j} t}{2}\right)
$$

The exponent in the above integral is just the quadratic form determined by the symmetric matrix, $\mathbf{B}-\mathbf{J}$, for which

$$
\operatorname{det}(\mathbf{B}-\mathbf{J})=\boldsymbol{\Delta}=\left(\Omega_{1} \Omega_{2}-1\right)^{2}=\frac{\cos \left(\frac{\omega_{1}+\omega_{2}}{2} t\right)}{\cos \left(\frac{\omega_{1}}{2} t\right) \cos \left(\frac{\omega_{2}}{2} t\right)},
$$

while the characteristic equation,

$$
\operatorname{det}(\mathbf{B}-\mathbf{J}-\lambda \mathbf{I})=\left[\left(\Omega_{1}-\lambda\right)\left(\Omega_{2}-\lambda\right)-1\right]^{2}=0,
$$

has double roots:

$$
\lambda_{ \pm}=-\frac{\Omega_{1}+\Omega_{2}}{2} \pm\left[1+\left(\frac{\Omega_{1}-\Omega_{2}}{2}\right)^{2}\right]^{\frac{1}{2}} .
$$

Hence, there are two alternatives for the overall change of phase increment (6.5),

(i) $\Theta=0$ if $\Omega_{1} \Omega_{2}<1$.

(ii) $\Theta= \pm \pi$ if $\Omega_{1} \Omega_{2}>1$.

The simplest case is that of a product of forward oscillations $\left(\omega_{1}\right.$ and $\left.\omega_{2}>0\right)$ such that neither has individually reached the caustic at $\omega_{j} t=\pi$, but $\left(\omega_{1}+\omega_{2}\right) t>\pi$. This is just case (ii), so that, if both $\omega_{j} t \approx \pi$, then $U_{t}(\mathbf{x}) \approx-1$.

For the Loschmidt echo, the returning motion is specified by $\omega_{2} \approx-\omega_{1}$, so that $\Omega_{2} \approx-\Omega_{1}$. For short times, both $\cos \left(\omega_{j} t / 2\right)>0$, so that $\Omega_{1} \Omega_{2}<0$, which is case (i). There is no new phase and we obtain $U_{t}(\mathbf{x}) \approx 1$. If one now allows $\omega_{1}$ to be slightly larger than $\pi$, though still $\left|\omega_{2} t\right|<\pi$, then $\Omega_{1}>0$ and $\Omega_{1} \Omega_{2}>1$, which is case (ii) and hence $\Theta=\pi$. But this exactly cancels the phase coming from $\cos \left(\omega_{1} t / 2\right)<0$, so that the overall sign of the propagator remains positive, just as for small times, that is, $U_{t}(\mathbf{x}) \approx 1$. Finally, if one allows both oscillators for the Loschmidt echo to pass their caustic, we are back in case (i) since $\Omega_{1} \Omega_{2}<0$. But now both amplitudes, $\cos \left(\omega_{j} t / 2\right)<0$, as long as $\left|\left(\omega_{1}+\omega_{2}\right) t\right|<\pi$, so the overall sign is still positive and, once again, $U_{t}(\mathbf{x}) \approx 1$.

The special simplicity of the harmonic oscillator allows for an appealing though untypical synthesis of these results, due to the identification of the previous conditions with the sign of $\boldsymbol{\Delta}^{1 / 2}$ in A.3.

(i) $\cos \left(\frac{\omega_{1}}{2} t\right) \cos \left(\frac{\omega_{2}}{2} t\right)>0$; then the sign coming from the integration is a minus if $\cos \left(\frac{\omega_{1}+\omega_{2}}{2} t\right)<0$, but this converts $\left|\cos \left(\frac{\omega_{1}+\omega_{2}}{2} t\right)\right|$ into $\cos \left(\frac{\omega_{1}+\omega_{2}}{2} t\right)$.

(ii) $\cos \left(\frac{\omega_{1}}{2} t\right) \cos \left(\frac{\omega_{2}}{2} t\right)<0$; then the integration sign is negative if $\cos \left(\frac{\omega_{1}+\omega_{2}}{2} t\right)>0$, which converts $\cos \left(\frac{\omega_{1}}{2} t\right) \cos \left(\frac{\omega_{2}}{2} t\right)<0$ into $\left|\cos \left(\frac{\omega_{1}}{2} t\right) \cos \left(\frac{\omega_{2}}{2} t\right)\right|$. 


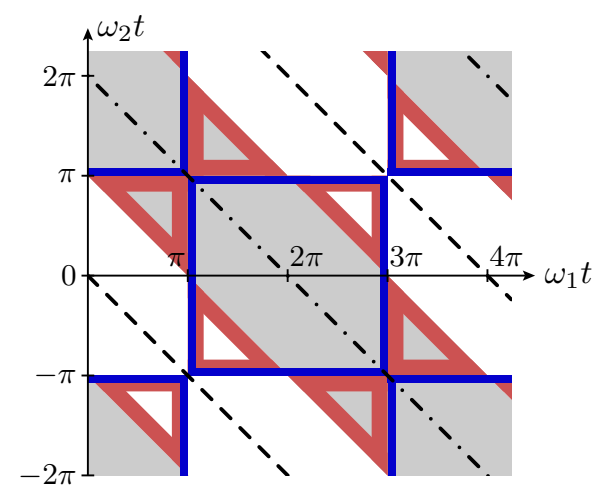

Figure A1. Overall sign for a product of harmonic oscillations: A negative sign arises within the blue (black) squares and within the red (dark grey) triangles, due to the sign of the product of the initial cosines or the crossing of a caustic, respectively. Together these generate a negative sign for the product propagator in the interior of the light grey stripes. The dashed lines correspond to the unit operator $\widehat{I}$, while all points on the dash-dotted lines correspond to $-\widehat{I}$.

In all cases the amplitude will be $\cos \left(\frac{\omega_{1}+\omega_{2}}{2} t\right)$ without any sign ambiguity, as portrayed in figure A1. One should be warned that this is a very special case: In general, one cannot evaluate the final phase without keeping detailed track of the intial phases and the phase increment!

For a Loschmidt echo, the product of oscillations as a function of time will define a vector in figure A1 that grows from the origin, nearly parallel to the dashed line. Thus, it will be a long time before it finally leaves the white strip and changes its sign. In contrast, the pair of oscillations in the same sense, that were first considered, runs in the direction of the other diagonal, so that the sign changes repeatedly as $t$ increases.

\section{Acknowledgments}

We thank Raul Vallejos, Eduardo Zambrano and Jiří Vaníček for stimulating discussions. AMOA thanks the hospitality of the University of Augsburg and, reciprocally, GLI is greatful to CBPF for its hospitality. Partial financial support from the National Institute for Science and Technology-Quantum Information, FAPERJ and $\mathrm{CNPq}$ (Brazilian agencies) is gratefully acknowledged.

\section{Bibliography}

[1] Ozorio de Almeida A M 1992 Proc. R. Soc. Lond. A 439139

[2] Ozorio de Almeida A M 1998 Phys. Rep. 295265

[3] Walls D F and Milburn G J 1995 Quantum Optics (Berlin: Springer)

[4] Toscano F, Vallejos R O and Wisniacki D 2009 Phys. Rev. E 80046218

[5] Kirchmair G, Vlastakis B, Leghtas Z, Nigg S E, Paik H, Ginossar E, Mirrahimi M, Frunzio L, Girvin S M and Schoelkopf R J 2013 Nature 495205

[6] Berry M V 1989 Proc. R. Soc. Lond. A 423219

[7] Maslov V P 1972 Théorie des Perturbations et Méthodes Asymptotiques (Paris: Dunod)

[8] Maslov V P and Fedoriuk M V 1981 Semi-Classical Approximation in Quantum Mechanics (Boston: Reidel)

[9] Gutzwiller M C 1990 Chaos in Classical and Quantum Mechanics (New York: Springer) 
[10] Creagh S C, Robbins J M and Littlejohn R G 1990 Phys. Rev. A 421907

[11] Robbins J M 1991 Nonlinearity 4343

[12] Bargmann V 1961 Comm. Pure Appl. Math. 14187

[13] Kramer P, Moshinsky M and Seligman T H 1975 in Group Theory and Its Applications vol III ed E M Loebl (New York: Academic Press)

[14] Guillemin V and Sternberg S 1984 Symplectic Techniques in Physics (Cambridge: Cambridge University Press)

[15] Voros A 1976 Ann. Inst. H. Poincaré (A) 2431

[16] Voros A 1977 Ann. Inst. H. Poincaré (A) 26343

[17] Littlejohn R G 1986 Phys. Rep. 138193

[18] de Gosson M 2006 Symplectic Geometry and Quantum Mechanics (Basel: Birkhäuser Verlag)

[19] Ozorio de Almeida A M, Vallejos R O and Zambrano E 2013 J. Phys. A: Math. Theor. 46 135304

[20] Dittrich T, Viviescas C and Sandoval L 2006 Phys. Rev. Lett. 96070403

[21] Ozorio de Almeida A M and Brodier O 2006 Ann. Phys., NY 3211790

[22] Dittrich T, Gomez E A and Pachon L A 2010 J. Chem. Phys. 132214102

[23] Arnold V I 1978 Mathematical Methods of Classical Mechanics (Springer: New York)

[24] Rivas A M F, Saraceno M and Ozorio de Almeida A M 2000 Nonlinearity 13341

[25] Williamson J 1936 Amer. J. Math. 58141

[26] Grossmann A 1976 Commun. Math. Phys. 48191

[27] Royer A 1977 Phys. Rev. A 15449

[28] Nicacio F, Maia R N P, Toscano F and Vallejos R O 2010 Phys. Lett. A 3744385

[29] paper in preparation 